\title{
Artisanat antique dans l'aire vésuvienne : le cas de la pierre
}

Campagne d'études 2015

Ghislain Vincent, Guilhem Chapelin, Marina Covolan, Émilie Grondin et Nicolas Leys

\section{(2) OpenEdition \\ Journals}

Édition électronique

URL : http://journals.openedition.org/cefr/1504

DOI : $10.4000 /$ cefr. 1504

ISSN : 2282-5703

Éditeur

École française de Rome

Référence électronique

Ghislain Vincent, Guilhem Chapelin, Marina Covolan, Émilie Grondin et Nicolas Leys, « Artisanat antique dans l'aire vésuvienne : le cas de la pierre ", Chronique des activités archéologiques de l'École française de Rome [En ligne], Les cités vésuviennes, mis en ligne le 01 mars 2016, consulté le 11 septembre 2019. URL : http://journals.openedition.org/cefr/1504; DOI : 10.4000/cefr.1504

Ce document a été généré automatiquement le 11 septembre 2019.

(c) École française de Rome 


\title{
Artisanat antique dans l'aire vésuvienne : le cas de la pierre
}

Campagne d'études 2015

\author{
Ghislain Vincent, Guilhem Chapelin, Marina Covolan, Émilie Grondin et \\ Nicolas Leys
}

\section{NOTE DE L'AUTEUR}

Nous tenons à remercier ici toutes les institutions et les personnes qui rendent possible cette étude.

1 Comme chaque année depuis $2013^{1}$, la mission s'est déroulée durant l'automne, pendant un mois, entre le 19 octobre et le 14 novembre 2015. Une phase de terrain a permis l'avancement de l'inventaire des blocs, de petit module en tuf gris, de colonnes et de piliers à demi-colonne engagée. Le nombre de blocs étudiés a ainsi été porté de 190 à 320 . La cartographie de leur localisation à l'échelle $1 / 10000^{\mathrm{e}}$ a été mise à jour (fig. 1). Cette année, pour la première fois, des blocs se trouvant à Herculanum ont été inventoriés. 
Fig. 1 - Carte de répartition des blocs dans Pompéi.

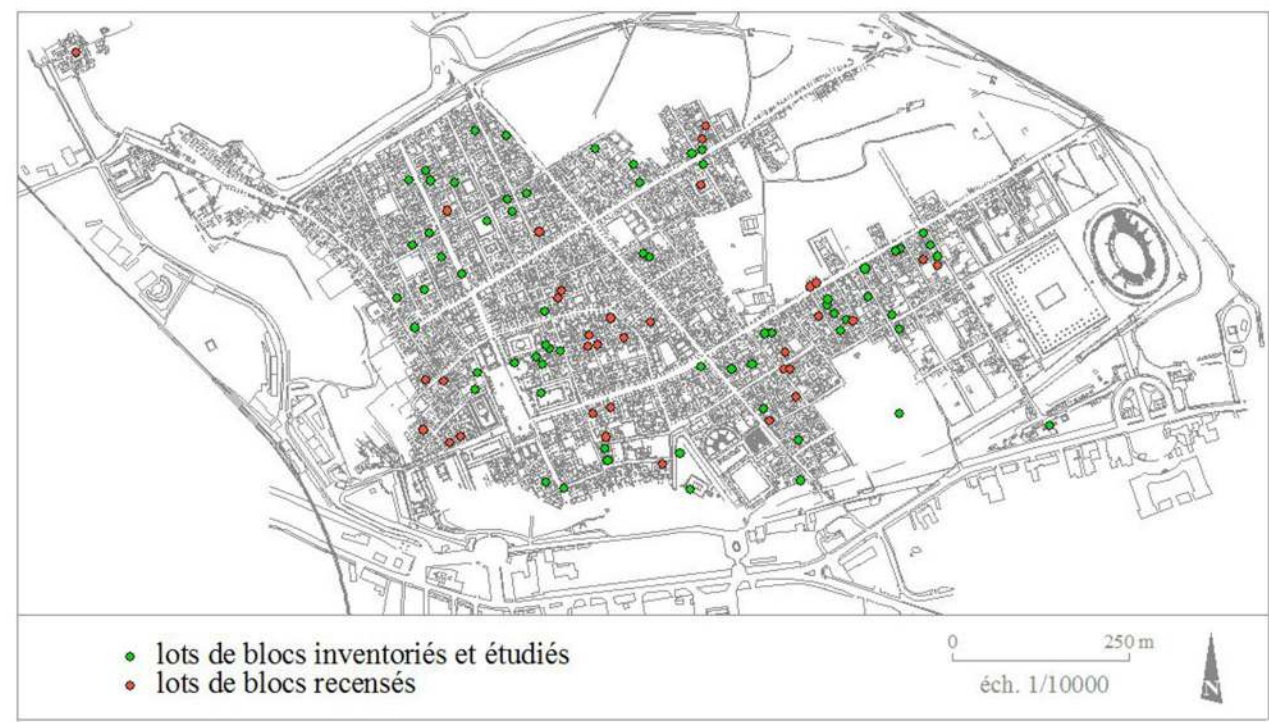

G. Chapelin, G. Vincent.

Malgré la réduction du nombre de zones accessibles, pour des raisons de sécurité, aux touristes et aux chercheurs par la mise en œuvre du Grande Progetto Pompei, nous avons pu accéder à l'essentiel des zones qui nous intéressaient.

\section{L'extraction et le transport de la pierre}

2 Un second volet a été consacré à la recherche, sur les territoires de Sorrente et Nocera (fig. 2), des restes de fronts de taille, même modernes, dans le but de localiser approximativement des points d'extraction possible de l'ignimbrite campanienne dans l'Antiquité. 
Fig. 2 - Affleurements d'ignimbrite campanienne grise et voies navigables.

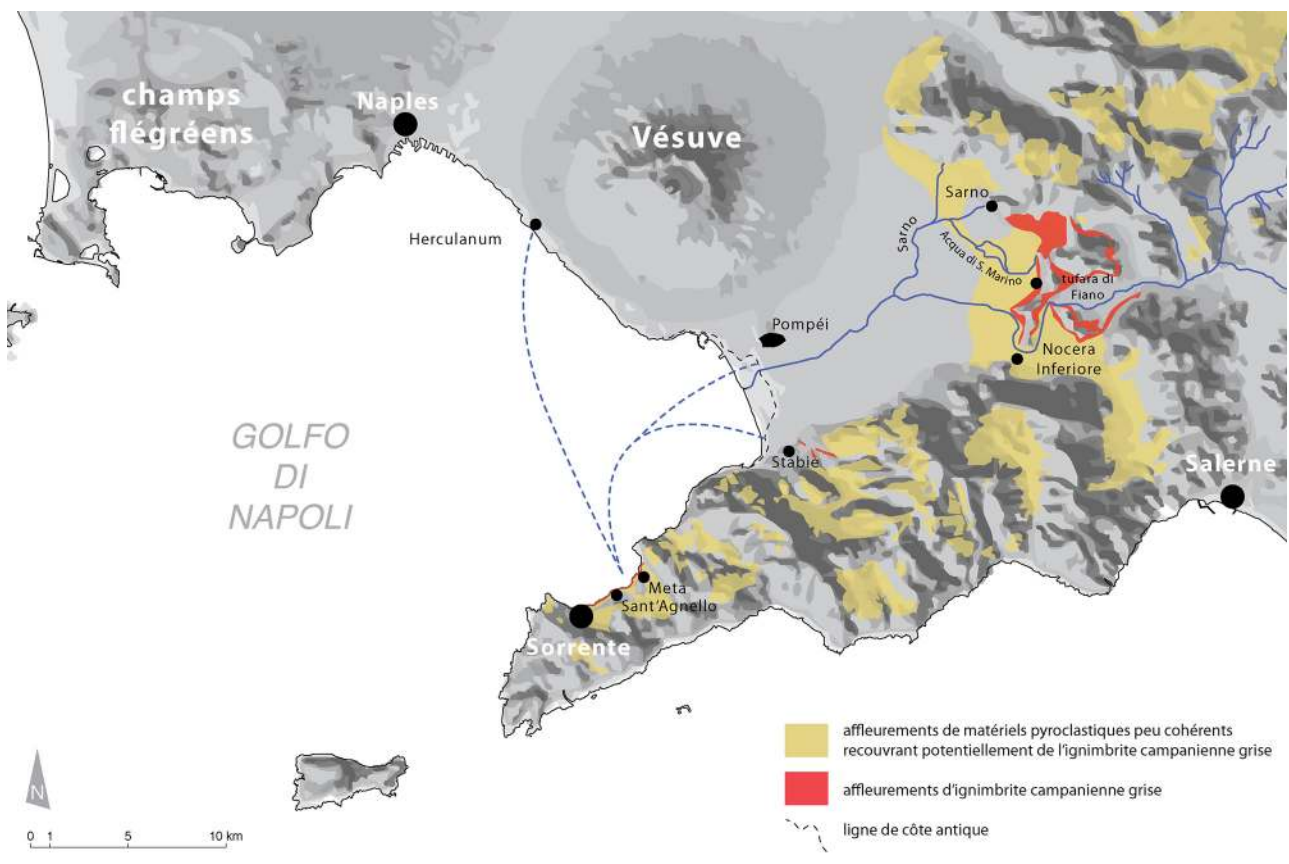

G. Chapelin, G. Vincent.

L'hypothèse de la présence de carrières antiques dans la région de Sarno qui approvisionneraient Pompéi par le biais du fleuve reste privilégiée (fig. 3).

Fig. 3 - Vue d'un front de taille «la tufara di Fiano».

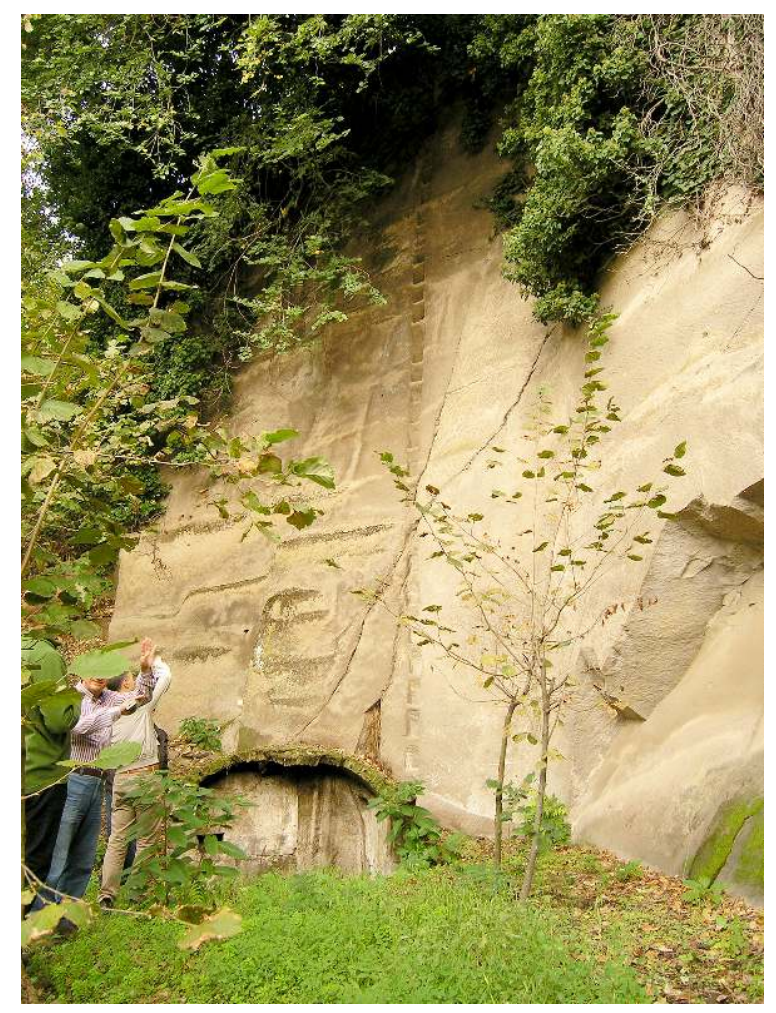

G. Chapelin, G. Vincent. 
D'une part, malgré un débit actuellement modeste, le fleuve est navigable, notamment par des barques à fond plat comme celle de Murecine ${ }^{2}$, et permet l'acheminement de matériaux lithiques. En effet, une profondeur de $1 \mathrm{~m}$ et une largeur de $5 \mathrm{~m}$ suffisent pour faire circuler des barques sur des cours d'eau dans le cadre du transport de matières premières lithiques ${ }^{3}$. Pour une barque d'une tonne environ, M. Pichon imagine, pour un cours d'eau du même gabarit que le Sarno situé en Touraine, une charge potentielle de trois tonnes de blocs ${ }^{4}$. Strabon précise en outre que, grâce au Sarno, Pompéi constituait un avant-port commode pour les villes de Nola, Nocera et Acerra (Strab., V, 4, 8). Ainsi, l'activité économique liée au fleuve est-elle déjà reconnue par le géographe antique.

D'autre part, si les carrières principales de tuf fournissant Pompéi avaient été installées sur la péninsule sorrentine, elles auraient aussi logiquement fourni très largement Herculanum.

Fig. 4 - Falaises de tuf gris de la côte sorrentine.

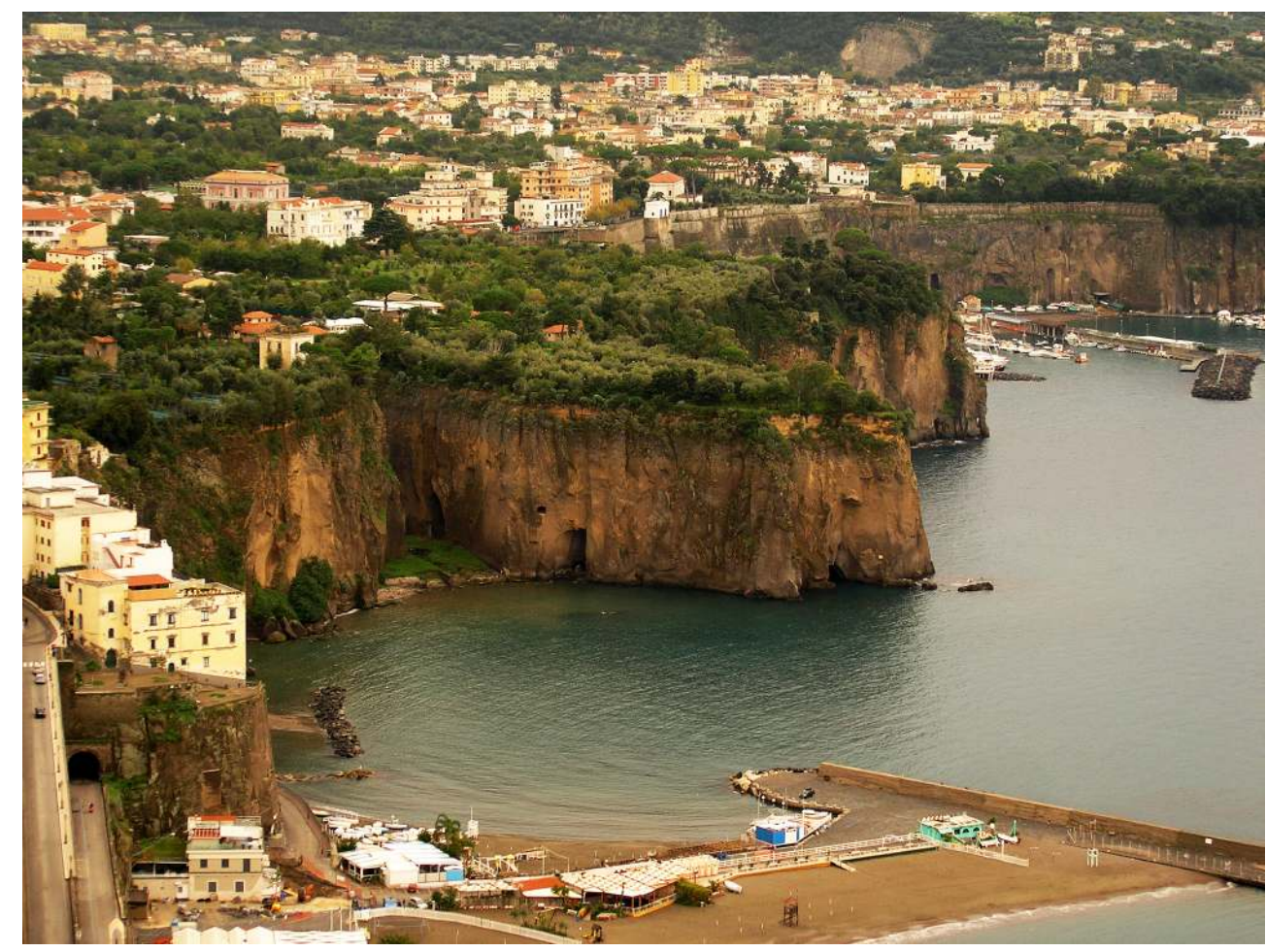

G. Chapelin, G. Vincent.

Or, proportionnellement aux surfaces mises au jour, le volume de tuf gris employé dans cette autre ville semble faible. Trois maisons seulement, emploient le tuf gris sous forme de colonnettes de premier étage (insula $V$, maison 5 : casa del mobilio carbonizzato, insula VI, maison 17: casa del Colonnato Tuscanico et insula V, maison 1: casa Sannitica). Contrairement à Pompéi, l'emploi du tuf jaune est prédominant. On en déduit que les approvisionnements étaient différents. Si l'ignimbrite campanienne a pu provenir occasionnellement de Sorrente, le phénomène reste probablement marginal au regard du volume de matériau employé durant la période samnite, et un peu après, sur le site de Pompéi. Enfin, l'emploi en très grande quantité de "calcaire du Sarno » dans le site révèle, s'il en était encore besoin, que le fleuve a servi à acheminer de gros et nombreux blocs. 


\section{Taille de la pierre}

Sur le plan technique, chaque catégorie distincte, colonne libre ou pilier à demi-colonne engagée, fait l'objet d'une opération de taille spécifique. Si la première fait systématiquement l'objet d'une approche au tour, ce n'est pas le cas de la seconde. Cette différence fondamentale s'explique aisément par l'aspect définitif de la pièce, cylindrique dans un cas, composée d'un parallélépipède uni à un demi cylindre dans l'autre. Ceci ne révèle pas une différence d'atelier mais plutôt une manière spécifique de répondre à des contraintes techniques. La chaîne opératoire, en l'état actuel de nos connaissances, peut être définie assez précisément pour chaque type d'éléments et nous travaillons à des schémas présentant chaque étape de travail depuis le bloc brut après extraction jusqu'à la mise en place des éléments dans la construction et leur finition.

\section{Expérimentation d'un tour à pierre horizontal}

Les blocs ayant appartenu à des colonnes, tant bases, fûts que chapiteaux, portent sur leurs lits des mortaises parallélépipédiques qui ont permis de les maintenir dans un tour à pierre.

Fig. 5 - Cavité axiale (a) de très petite dimension (51) et mortaise (b) de tour courante (300).

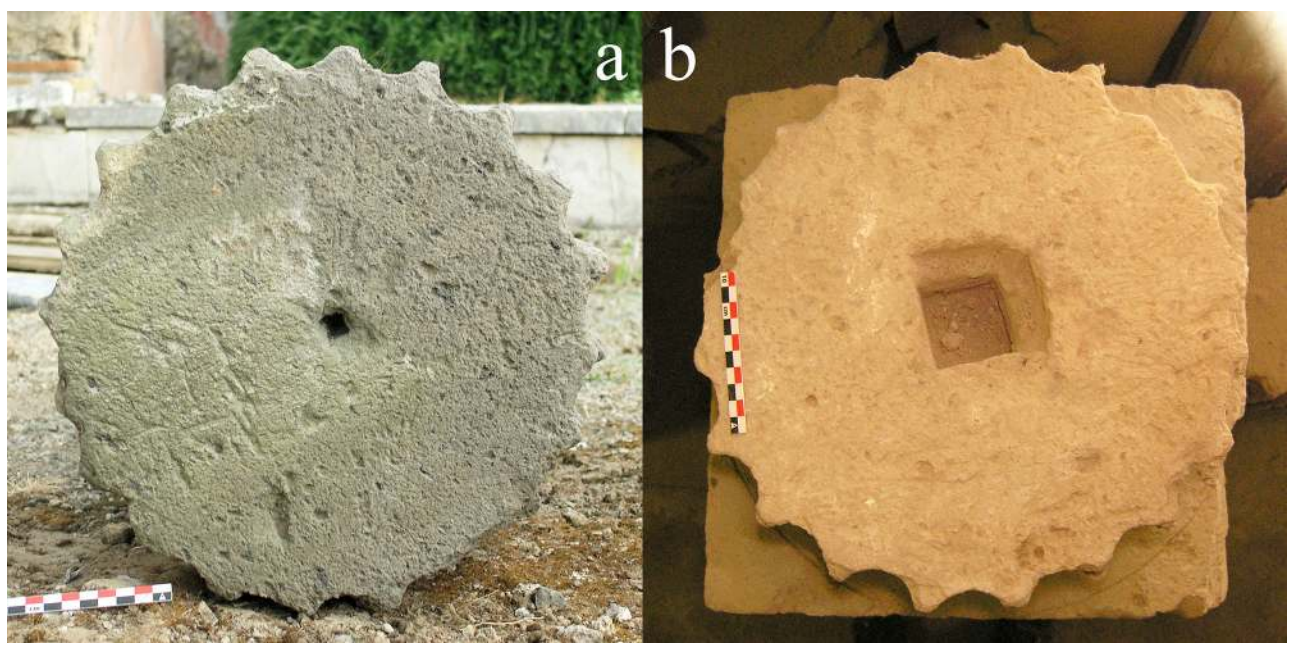

G. Chapelin, G. Vincent.

Dans deux cas seulement des cavités axiales très petites, qu'on ne peut plus appeler mortaises, empêchent qu'il s'agisse d'un tour horizontal (fig. 5a). Pour une écrasante majorité des blocs on doit envisager une machine à axe aussi bien vertical qu'horizontal (fig. 5b). Depuis le début de notre étude, il nous a semblé que l'orientation verticale était la plus simple et la plus efficace pour l'axe d'un tour à pierre. Un magnifique exemple de tour vertical manuel a été réalisé et testé en Poitou-Charentes ${ }^{5}$. Bien que cette expérience valide parfaitement la possibilité que des tours verticaux aient été utilisés dans l'Antiquité, l'hypothèse d'un tour horizontal nous a semblé plausible. L'idée est née de l'observation des traces de tournage sur des faces opposées comme le lit d'attente d'un chapiteau pour faire ressortir le scamillus et la sous-face du chapiteau pour le sillon prolongeant le fût pour la préparation de chapiteaux ioniques à quatre faces. Le 
positionnement du bloc sur un axe vertical obligerait le tourneur à faire une de ces opérations par en dessous, ce qui n'est pas impossible mais l'oblige à adopter une position inconfortable, ou alors demanderait de retourner le bloc, ce qui est faisable mais peu pratique. Ce problème ne se pose pas si l'axe est horizontal. Le tour horizontal, tel que nous l'avons réalisé pour une expérimentation (fig. 6), présente tout d'abord l'avantage d'aider à soulever le bloc facilement par un simple effet de levier (fig. 7) et ainsi à tourner des blocs de poids considérables sans avoir à les manipuler et les équilibrer sur le tour.

Fig. 6 - Prototype de tour horizontal.

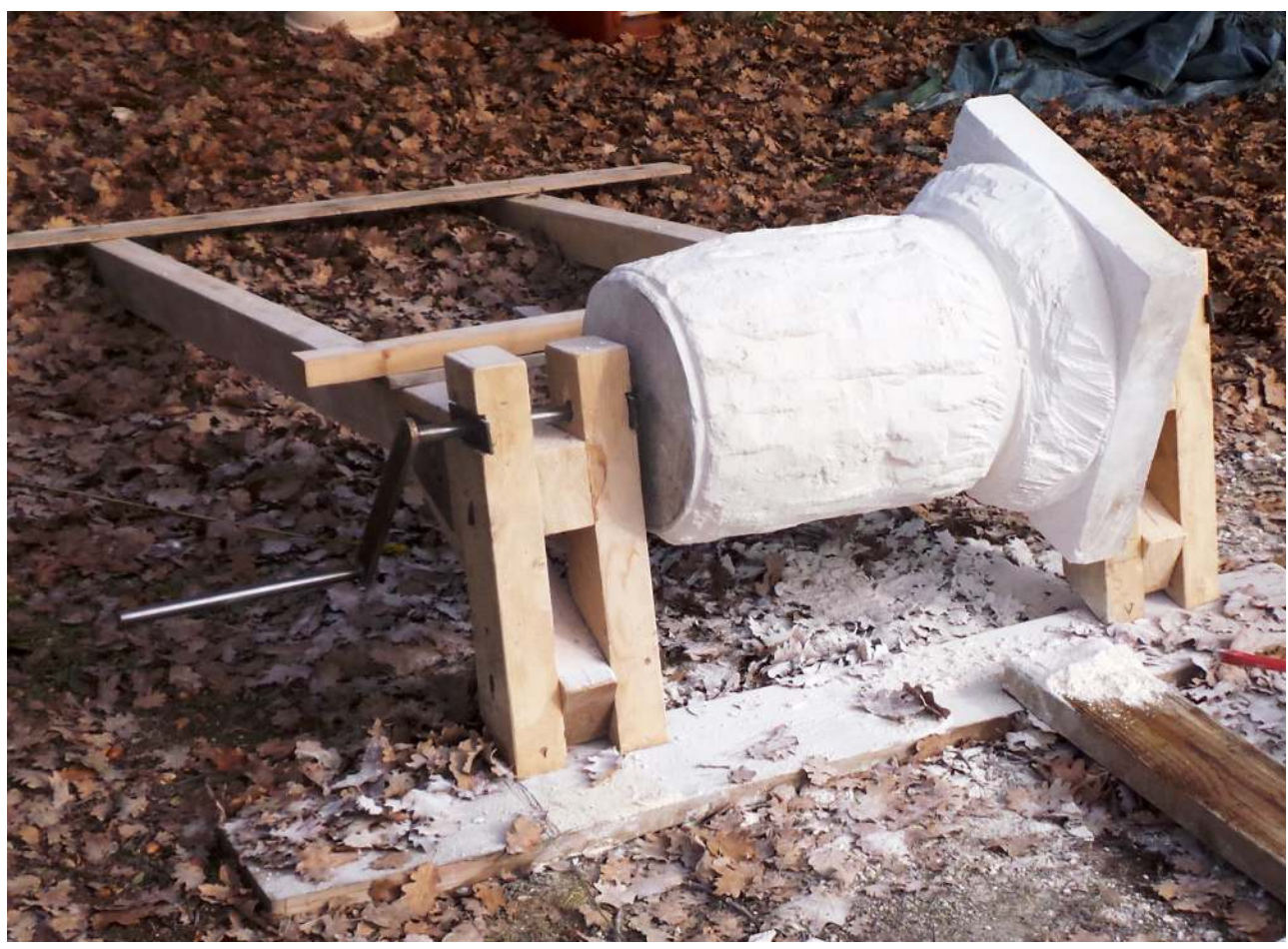

G. Chapelin.

Fig. 7 - Principe de basculement du tour permettant de soulever le bloc.

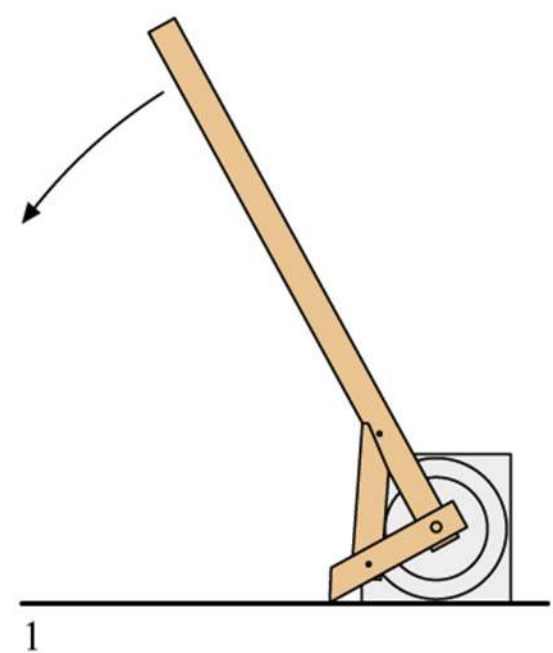

G. Chapelin.

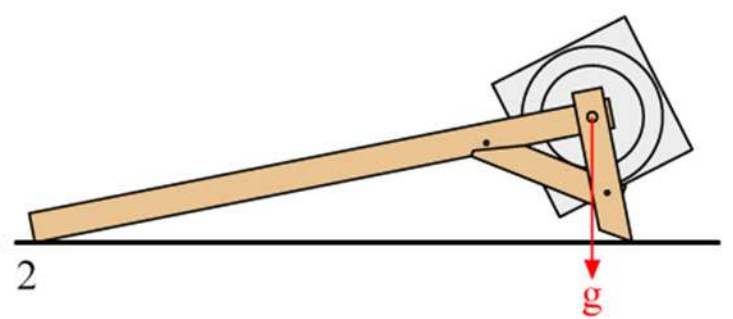

g 
Il suffit de pratiquer les deux mortaises sur deux faces opposées du bloc, alors que celui-ci est à terre, d'y loger les deux extrémités de l'axe du tour et de faire basculer le tout. Ce basculement a pour effet de décoller le bloc du sol. Une fois les longues pièces de bois à terre, la projection au sol du centre de gravité $(\mathrm{g})$ du bloc est située entre les points d'appui du tour, donc à l'intérieur de la surface de sustentation de l'ensemble qui est alors stable. Des pièces de bois transversales clouées permettent de rigidifier le tout. On peut alors mettre en mouvement le bloc au moyen d'une manivelle. Bien qu'une faible vitesse de rotation n'empêcherait pas le tournage de la pierre, il est possible en procédant ainsi d'atteindre sans grand effort des vitesses de rotations de l'ordre de 30 à 40 tours par minute pour un bloc de $280 \mathrm{~kg}$. Afin d'éprouver le mécanisme, l'expérience a été faite jusqu'ici avec un calcaire coquillier fin de Provence, la pierre d'Oppède (Vaucluse) d'une masse volumique bien supérieure à celle de l'ignimbrite campanienne. Pour compléter l'expérience nous souhaiterions nous procurer du tuf gris de Nocera et vérifier s'il est possible de reproduire à l'identique les blocs pompéiens.

\section{Un ordre dorique au premier étage?}

On ne compte pas les cas d'imposants ordres doriques situés au rez-de-chaussée de maisons samnites pompéiennes comme, à titre d'exemple, celle de l'atrium corinthien de la casa dei Diadumeni (IX, 1, 20). En revanche, nous ne connaissons que deux cas d'emploi de l'ordre dorique à l'étage ce qui constitue une curiosité au regard de la superposition «naturelle » des ordres. Un premier lot de blocs de piliers à demi-colonnes engagées est entreposé dans la rue séparant les insulae IX, 8 et IX, 9. Il semble provenir d'un premier étage compte tenu de ses dimensions relativement faibles. Un second lot dans la maison I, 13, 8 regroupe des colonnettes aux dimensions très voisines de celles de tous les lots de colonnettes ioniques. Les chapiteaux sont pourtant doriques et aucune base n'est présente. Ces derniers révèlent la présence non loin d'une colonnade d'étage de cinq colonnes au moins. L'inachèvement des fûts se déduit de la présence de traces de tournage sur la surface cylindrique au contact des lits entre lesquelles l'épiderme de la pierre porte encore les traces de dégrossissage au marteau taillant.

Fig. 8 - Chapiteau 203 (a), exemple de chapiteau dorique provenant de la maison I, 13, 8 et détail de la mouluration (b).

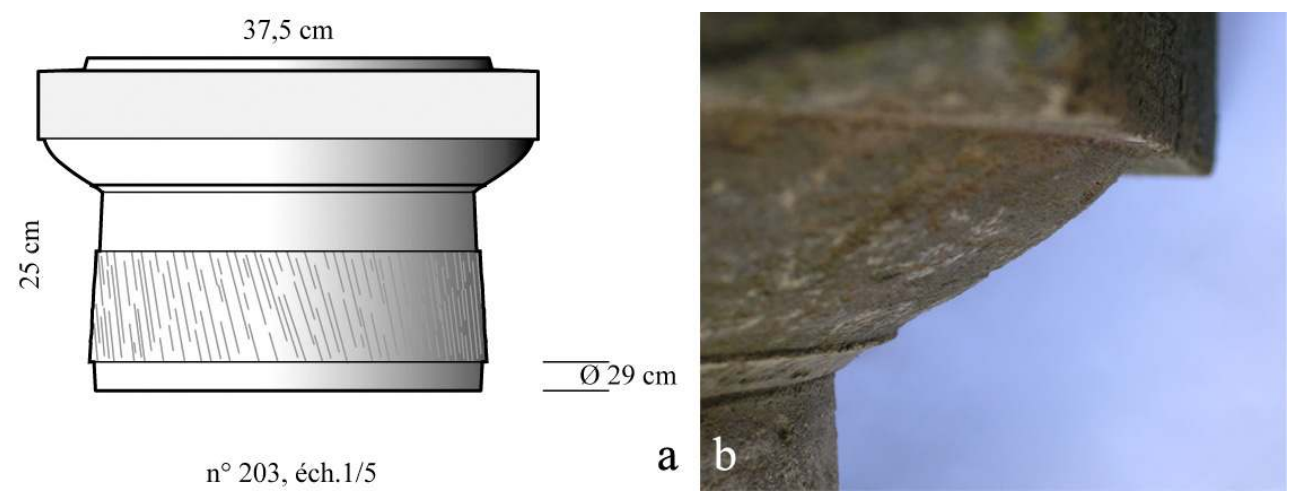

G. Chapelin, G. Vincent.

La très subtile mouluration des chapiteaux, entièrement tournée, est composée d'une toute petite doucine droite, d'un minuscule filet et d'une échine à la courbure à peine sensible (fig. 8). 


\section{Des chapiteaux corinthiens italiques}

7 Les chapiteaux corinthiens de petit module de style italique à acanthes en feuilles de choux sont assez rares en raison d'un emploi particulier comme pour des œecus ou peutêtre des laraires. Deux chapiteaux très semblables ont été inventoriés en 2015 l'un dans le dépôt des Granai et l'autre dans la nécropole de la Porta Nocera (respectivement $\mathrm{n}^{\circ} 201$, inv. Sap : 69693) (fig. 9) et 296 de notre inventaire.

Fig. 9 - Chapiteau corinthien italique partiellement tourné.

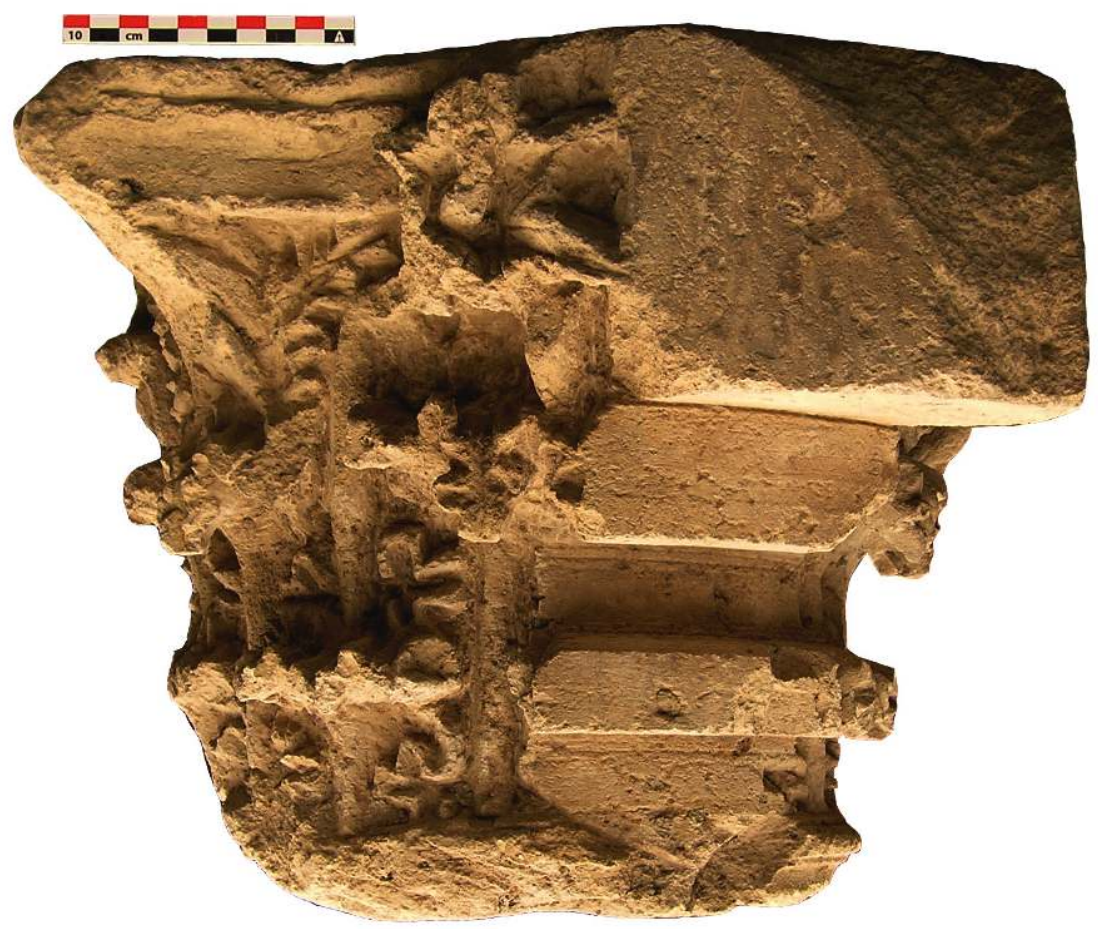

Inv. Sap 69693, n²01.

G. Chapelin, G. Vincent.

Ces deux blocs sont d'un grand intérêt car leur décoration sculptée ne couvre pas toute sa périphérie et ils montrent en détail les proportions du volume façonné lors d'une étape du tournage. Le geste technique est semblable à celui de la taille des autres chapiteaux où un volume parallélépipédique est laissé au sommet du bloc et la partie basse est tournée.

\section{BIBLIOGRAPHIE}

Andreau 1994 = J. Andreau, Affaires financières à Pouzzoles au I Ir s. ap. J.-C. : les tablettes de Murecine, dans REL, 72, 1994, p. 39-55. 
Gaillard 2008 : J. Gaillard, La pierre dans la Saintonge antique et médiévale, dans ADLFI. Archéologie de la France - Informations [En ligne], Poitou-Charentes, mis en ligne le 01 mars 2008. URL : http:// adlfi.revues.org/1260

Pichon 2002 : M. Pichon, Le transport par voie navigable : l'exemple du site de Tendu (Indre), dans Gallia , 59, 2002, p. 83-88.

\section{NOTES}

1. https://cefr.revues.org/1166; https://cefr.revues.org/1284.

2. Andreau 1994, p. 39.

3. Pichon 2002.

4. Pichon 2002, p. 83-88.

5. Gaillard 2008.

INDEX

Index géographique : Herculanum, Pompéi

Mots-clés : Artisanat, taille de pierre, Pompéi, tour à pierre, chapiteau, colonne, pilier, étage institutions Inrap, Centre Jean Bérard, École française de Rome, Soprintendenza Pompei, Ministère des Affaires étrangères et du développement international (Paris)

\section{AUTEURS}

\section{GHISLAIN VINCENT}

Inrap, Archéologie des Sociétés Méditerranéennes (UMR 5140) - ghislain.vincent[at]inrap.fr

\section{GUILHEM CHAPELIN}

Centre Jean Bérard (USR 3133 CNRS-EFR) - guilhem.chapelin[at]laposte.net

\section{MARINA COVOLAN}

Università degli Studi di Padova - marina2789[at]hotmail.it

\section{ÉMILIE GRONDIN}

Université de Lyon - emilie.grondin[at]univ-lyon2.fr

\section{NICOLAS LEYS}

Université de Paris IV-Sorbonne - leysnicolas[at]gmail.com 